\title{
LOCALIZATION AND SHEAF REFLECTORS
}

\author{
BY
}

\section{J. LAMBEK AND B. A. RATTRAY(1)}

\begin{abstract}
Given a triple $(S, \eta, \mu)$ on a category $A$ with equalizers, one can form a new triple whose functor $Q$ is the equalizer of $\eta S$ and $S \eta$. Fakir has studied conditions for $Q$ to be idempotent, that is, to determine a reflective subcategory of $A$. Here we regard $S$ as the composition of an adjoint pair of functors and give several new such conditions. As one application we construct a reflector in an elementary topos $A$ from an injective object $I$, taking $S=I^{I^{(-)}}$. We show that this reflector preserves finite limits and that the sheaf reflector for a topology in $A$ can be obtained in this way. We also show that sheaf reflectors in functor categories can be obtained from a triple of the form $S=I^{(-, I)}, I$ injective, which we studied in a previous paper. We deduce that the opposite of a sheaf subcategory of a functor category is tripleable over Sets.
\end{abstract}

1. Adjoint pairs and reflectors. Throughout this section $A$ will be a category with equalizers, $(S, \eta, \mu)$ a triple (standard construction, monad) on $A$ and $\kappa: Q \rightarrow S$ the equalizer of $S \eta$ and $\eta S$. There is a unique triple $\left(Q, \eta_{1}, \mu_{1}\right)$ such that $k$ is a map of triples. This process of constructing one triple from another has been studied by Applegate and Tierney [2] and Fakir [6]. Fakir gave several necessary and sufficient conditions for $Q$ to be idempotent, that is, for $\eta_{1} Q: Q \rightarrow Q^{2}$ to be an equivalence.

Let Fix $Q$ be the full subcategory of A consisting of those objects $A$ of A for which $\eta_{1}(A)$ is an isomorphism, that is, $\eta(A)$ is the equalizer of $\eta S(A)$ and $S \eta(A)$. For each $A$ in Fix $Q$ we choose $\kappa(A)=\eta(A)$ so that $\eta_{1}(A)$ is the identity. Clearly, $Q$ is idempotent if and only if Fix $Q$ coincides with the full image $\operatorname{Im} Q$ of $Q$. In view of [1, p. 233], $Q$ is idempotent if and only if Fix $Q$ is a reflective subcategory of $A$ with reflection maps $\eta_{1}(A): A \rightarrow Q(A)$.

We shall take $S=U F$, where $A \stackrel{F}{\longrightarrow} B \stackrel{U}{\longrightarrow} A$ is a pair of functors with $F$ left adjoint to $U$. As is well known, if $(U, F)$ is such an adjoint pair with adjunctions $\eta: 1 \rightarrow U F$ and $\epsilon: F U \rightarrow 1$, then $(U F, \eta, U \epsilon F)$ is a triple; con-

Received by the editors July 2, 1973.

AMS (MOS) subject classifications (1970). Primary 18A40; Secondary 18F20, $18 \mathrm{C} 15,18 \mathrm{D} 15$.

Key words and phrases. Localization, triple, reflective subcategory, injective, sheaf, cartesian closed category, topos.

(1) One author held a research grant from the N.R.C. of Canada, the other enjoyed the hospitality of the E.T.H. in Zürich in 1972-1973, while this paper was being written. 
versely, every triple has this form [11, p. 136]. We shall obtain several conditions in terms of $U$ and $F$ equivalent to $Q$ being idempotent. $\left({ }^{2}\right)$

We denote the limit closure of a subcategory $B$ of $A$ by $L(B)$. We recall that this is the smallest full replete limit closed subcategory of $A$ containing $B$. We also recall that, for any class $M$ of maps of $A$, an object $A$ of $A$ is $M$-injective if $(m, A)$ is a surjection for all $m \in M$.

Lemma 1.1. Im $U \subseteq$ Fix $Q$.

Proof. We recall that $U \epsilon(B) \eta U(B)=1_{U(B)}$ for all $B$ in $B[11$, p. 80]. Now, if $\eta(A): A \rightarrow S(A)$ has a left inverse $p$ for some $A$ in $A$, then $p$ and $S(p)$ make the canonical diagram $A \longrightarrow S(A) \Longrightarrow S^{2}(A)$ into a split equalizer diagram. Thus, $\eta U(B)$ coincides with $\kappa U(B)$, and so $U(B) \in$ Fix $Q$ for all $B$ in $B$.

PROPOSITION 1.2. The following statements are equivalent:

(a) $Q$ is idempotent, that is, it induces a reflector $\mathrm{A} \rightarrow \operatorname{Fix} Q$.

(b) Fix $Q=L(\operatorname{Im} U)$, the limit closure of the full image of $U$.

(c) An object $A$ of $A$ is in Fix $Q$ if and only if there is an equalizer diagram $A \rightarrow U(B) \Longrightarrow U\left(B^{\prime}\right)$ in $A$ for some $B, B^{\prime}$ in $B$.

(d) $U(B)$ is $\kappa(A)$-injective for each $A$ in $A$ and $B$ in $B$.

(e) $F K(A)$ is a split mono, that is, has a left inverse, for each $A$ in $A$.

(f) $F_{K}(A)$ is a mono for each $A$ in $A$.

REMARK. Fakir [6] showed that (a) is equivalent to

(g) $U F_{K}(A)$ is mono for each $A$ in $A$.

We use $(\mathrm{g}) \Rightarrow(\mathrm{a})$ in our proof. Later we shall obtain Proposition 1.5, from which we can deduce the equivalence of (a)-(e) (not, however, (f)) without using Fakir's result.

Proof. Let $E$ be the full subcategory of those objects $A$ of $A$ for which there exists an equalizer diagram $A \rightarrow U(B) \Longrightarrow U\left(B^{\prime}\right)$ for some $B, B^{\prime}$ in $B$. Then

Fix $Q \subseteq \operatorname{Im} Q \subseteq E \subseteq L(\operatorname{Im} U \subseteq L($ Fix $Q)$,

the last inclusion following from Lemma 1.1.

Suppose (a) or (b), then Fix $Q$ is limit closed, hence all inclusions become equalities, in particular (b) and (c) hold. Suppose (c), then Fix $Q=\operatorname{Im} Q$ hence (a) holds.

Thus, (a), (b) and (c) are equivalent. We now show that (a) and (b) imply (d). We are given that $Q$ induces a reflector onto $L(\operatorname{Im} U)$, and we wish to show

(2) we are indebted to W. Spears for pointing out that the proof of Lemma 1.1, which we had considered only in the special case of $\S 2$, actually works in general. 
that every map $u: Q(A) \rightarrow U(B)$, for any $B$ in $B$, factors through $\kappa(A)$. Indeed, let $v: F(A) \rightarrow B$ correspond to $u \eta_{1}(A): A \rightarrow U(B)$ under the isomorphism $A(A, U(B)) \rightarrow B(F(A), B)$, then

$$
U(v) \kappa(A) \eta_{1}(A)=U(v) \eta(A)=u \eta_{1}(A),
$$

hence $U(v) \kappa(A)=u$, since $\eta_{1}(A)$ is a reflection map.

Clearly, (e) $\Rightarrow(f)$ and $(f) \Rightarrow(g)$. We refer to Fakir [6] for the implication $(\mathrm{g}) \Rightarrow(\mathrm{a})$. Finally, the equivalence of $(\mathrm{d})$ and $(\mathrm{e})$ is a consequence of

Lemma 1.3. Let $m: A \rightarrow A^{\prime}$ be a map in $A$. Then $F(m)$ is a split mono if and only if $U(B)$ is m-injective for each $B$ in $B$.

PRoOF. $U(B)$ is $m$-injective if and only if $A(m, U(B)): A\left(A^{\prime}, U(B)\right) \rightarrow$ $A(A, U(B))$ is a surjection. In view of the natural isomorphism $A(-, U(B)) \rightarrow$ $B(F(-), B)$, this is so if and only if $B(F(m), B): A\left(F\left(A^{\prime}\right), B\right) \rightarrow A(F(A), B)$ is a surjection. But this means that every map $F(A) \rightarrow B$ factors through $F(m)$, that is to say, that $F(m)$ is a split mono, as is seen from the special case of the identity map $F(A) \rightarrow F(A)$.

Proposition 1.4. Let $M$ be a class of maps in A which contains $\kappa(A)$ for each $A$ in $A$, and suppose that $U(B)$ is $M$-injective for each $B$ in $B$. Then $Q$ induces a reflector $A \rightarrow L(\operatorname{Im} U)$ which carries all elements of $M$ into regular monos of $L(\operatorname{Im} U)$.

PRoof. That $Q$ induces a reflector onto the subcategory is clear from Proposition 1.2. Let $m: A \rightarrow A^{\prime}$ be in $M$, then $F(m)$ is a split mono by Lemma 1.3 , hence so is $S(m)=U F(m)$. Consider the diagram

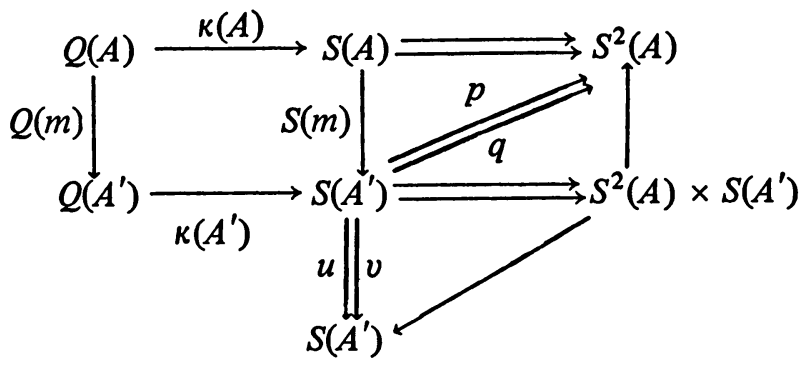

By definition, $k(A)$ is the equalizer of the two canonical maps $S(A) \rightarrow S^{2}(A)$. Since $S(m)$ is a split mono, these may be extended to maps $p, q: S\left(A^{\prime}\right) \rightarrow$ $S^{2}(A)$. Moreover, the split mono $S(m)$ is the equalizer of two maps $u, v$ : $S\left(A^{\prime}\right) \rightarrow S\left(A^{\prime}\right)$. Therefore, $\kappa\left(A^{\prime}\right) Q(m)=S(m) \kappa(A)$ is the equalizer of the pair of maps $\langle p, u\rangle,\langle q, v\rangle: S\left(A^{\prime}\right) \rightarrow S^{2}(A) \times S\left(A^{\prime}\right)$. Since $k\left(A^{\prime}\right)$ is mono, we con- 
clude that $Q(m)$ is the equalizer of the pair of maps $\langle p, u\rangle_{\kappa}\left(A^{\prime}\right),\langle q, v\rangle_{\kappa}\left(A^{\prime}\right)$ in $L(\operatorname{Im} U)$.

We shall obtain a new description of $Q$ which relates it more closely to $\operatorname{Im} U$.

Proposition 1.5. For all $A$ in $A, \kappa(A): Q(A) \rightarrow S(A)$ is the joint equalizer of all pairs of maps $r, s$ from $S(A)$ to an object in $\operatorname{Im} U$ such that $r \eta(A)=$ $s \eta(A)$.

Proof. Consider any map $r: S(A) \rightarrow U(B)$, then $r \eta(A): A \rightarrow U(B)$ gives rise to $r^{*}: F(A) \rightarrow B$, where $r^{*}=\epsilon(B) F(r(A))$. A routine calculation shows that $r k(A)=U\left(r^{*}\right) \kappa(A)$; for, if $r^{\prime}: F S(A) \rightarrow B$ corresponds to $r: S(A) \rightarrow U(B)$, then $U\left(r^{\prime}\right) \eta S(A)=r$ and $U\left(r^{\prime}\right) S \eta(A)=U\left(r^{*}\right)$.

Now suppose that $r, s: S(A) \rightarrow B$ are such that $r \eta(A)=s \eta(A)$, then $r^{*}=$ $s^{*}$, hence $r k(A)=\operatorname{sk}(A)$. Since $\kappa(A)$ is the equalizer of one such pair, namely of the canonical maps $S(A) \rightarrow S^{2}(A)$, it follows that $\kappa(A)$ is the joint equalizer of all such pairs.

Using Proposition 1.5 we can give an alternative proof that $(d) \Rightarrow(a)$ in Proposition 1.2, thus obtaining a self-contained proof of the equivalence of the conditions (a) to (e).

Assume that $U(B)$ is $\kappa(A)$-injective for all $A$ in $A$ and $B$ in $B$. We claim that $\eta_{1}(A): A \rightarrow Q(A)$ is a reflection map from $A$ to $L(\operatorname{Im} U)$. One easily sees that this amounts to showing that for each $f: A \rightarrow U(B)$ there exists a unique $f^{\prime}: Q(A) \rightarrow U(B)$ such that $f^{\prime} \eta_{1}(A)=f$. The existence is clear, since $f^{\prime}=U \epsilon(B) S(f) \kappa(A)$ is one such map. Suppose $f^{\prime} \eta_{1}(A)=f^{\prime \prime} \eta_{1}(A)$. Since $U(B)$ is $\kappa(A)$-injective, there exist $g^{\prime}, g^{\prime \prime}: S(A) \rightarrow U(B)$ such that $g^{\prime} k(A)=f^{\prime}$ and $g^{\prime \prime} k(A)=f^{\prime \prime}$. Then $g^{\prime} \eta(A)=g^{\prime \prime} \eta(A)$, hence $f^{\prime}=g^{\prime} k(A)=g^{\prime \prime} k(A)=f^{\prime \prime}$ by Proposition 1.5. Since $\operatorname{Im} Q \subseteq L(\operatorname{Im} U), \eta_{1}(A)$ is also a reflection map to $\operatorname{Im} Q$. It easily follows that $\eta_{1} Q(A)$ is an isomorphism.

As a corollary to Proposition 1.2 we obtain the following generalization of the dual of [9, Proposition 2].

Proposition 1.6. Each of the following conditions implies the next:

(a) $Q$ is canonically isomorphic to the identity, that is, $\eta_{1}$ is a natural isomorphism.

(b) For each object $A$ of $A$ there is an object $B$ of $B$ and a regular monomorphism $A \rightarrow U(B)$.

(c) For each object $A$ of $A$ there are objects $B$ and $B^{\prime}$ and an equalizer diagram $A \rightarrow U(B) \Longrightarrow U\left(B^{\prime}\right)$.

(d) $A$ is the limit closure of the full image of $U$. 
Moreover, (d) $\Rightarrow(\mathrm{a})$ if $U(B)$ is $\kappa(A)$-injective for each $B$ in $B$ and $A$ in $A$.

We shall consider a number of examples to which Proposition 1.2 may be applied.

EXAMPLE 1.7. Let $A$ be any complete category, $B=$ Sets $^{\circ \mathrm{p}}, U=I^{(-)}$ and $F=(-, I)$, where $I$ is a fixed object of $A$. This situation has been studied at length in [9].

ExAmPLE 1.8. Let $A=\operatorname{Mod} R, B=\operatorname{Mod} S,{ }_{R} C_{S}$ a given bimodule, $U=\operatorname{Hom}_{S}(C,-), F=(-) \otimes_{R} C$. Then $F(\kappa(A))$ is mono if ${ }_{R} C$ is flat (more generally, if it is $\kappa$-flat, analogous to $k$-injective in [9]). This example suggests a connection with Ulmer's work [17]. The subfunctor $Q$ of $U F$ can be calculated as follows. Let $\eta(A): A \rightarrow U F(A)$ be the canonical map defined by $\eta(A)(a)(c)=a \otimes c$ for $a \in A$ and $c \in C$. Take any element $s \in U F(A)$, then $s \in Q(A)$ if and only if, for all $c \in C,(\eta(A) \otimes 1)(s(c))=s \otimes c$. This example may be extended to allow $B$ to be any additive category, provided $C$ is an object of $B$ and there is given a homomorphism of $R$ into the ring of endomorphisms of $C$.

EXAMPLE 1.9. Let there be given a topological space $V$ with the underlying set $P$ of points. Both $V$ and $P$ may be regarded as small categories: the objects of $V$ are open sets, the maps of $V$ are inclusions; moreover, $P=P$ op is a discrete category. We take $A=$ Sets ${ }^{\text {op }}$ and $B=$ Sets $^{\text {pop }}$, and define the stalk functor $F: A \rightarrow B$ and the (discontinuous) sections functor $U: B \rightarrow A$ by

$$
F(A)(P)=\varliminf_{P \in V} A(V), \quad U(B)(V)=\prod_{P \in V} B(P) .
$$

It is easily seen that $F$ is left adjoint to $U$. Van Osdol [19] showed that the localization functor $Q$ determined by this adjoint pair is the functor which associates to each presheaf $A$ its associated sheaf. To apply Proposition 1.2, one wants to show that $F$ preserves regular monos. In fact, more is true, $F$ preserves finite limits, because in Sets filtered colimits preserve finite limits. From this one may easily deduce that $Q$ preserves finite limits.

This example may be generalized in two directions. Clearly, the same argument applies when the category of sets is replaced by any complete category with filtered colimits that preserve finite limits. On the other hand, one can replace the topological space $V$ by any small category equipped with a Grothendieck topology, at least when there are enough points. However, the results to be obtained by this method have been anticipated by Ulmer [18]

The functor $Q$ which appears in Proposition 1.4 may be called the localization functor determined by the adjoint pair $(F, U)$. 
2. Injectives in an elementary topos. We turn to the construction of the sheaf reflector in an elementary topos which was announced in [9, Example 4].

For the moment, let $A$ be a cartesian closed category, that is, a category equipped with finite products so that, for each object $A$, the functor $A \times(-)$ : $\mathrm{A} \rightarrow \mathrm{A}$ has a right adjoint $(-)^{A}$. It is easily seen that, for any object $I$ of $A$, the functor $I^{(-)}: A^{\text {op }} \rightarrow A$ has the left adjoint $I^{(-)}: A \rightarrow A^{\text {op }}$. This adjoint pair determines a triple $(S, \eta, \mu)$ on $A$, with $S(A)=I^{\left(I^{A}\right)}$. Assuming that $A$ has equalizers, we obtain $Q$ from $S$ as in $\S 1$.

PROPOSITION 2.1. Let A be a cartesian closed category with equalizers, $I$ an object of $\mathrm{A}, S$ and $Q$ as above. Thein the following statements are equivalent:

(a) $Q$ is idempotent, that is, it induces a reflector $A \rightarrow$ Fix $Q$.

(b) Fix $Q=L\left(\left\{I^{A} \mid A \in A\right\}\right)$.

(c) An object $A$ of $A$ is in Fix $Q$ if and only if there is an equalizer diagram $A \rightarrow I^{B} \Longrightarrow I^{B^{\prime}}$ in $A$ for some $B, B^{\prime}$ in $A$.

(d) $I^{B}$ is $\kappa(A)$-injective for all $A$ and $B$ in $A$.

If $I$ is injective with regard to all monos (all regular monos) then these conditions are satisfied and the reflector takes all monos (all regular monos) of A to regular monos of $L\left(\left\{I^{A} \mid A \in A\right\}\right)$.

Proof. This is an immediate consequence of Propositions 1.2 and 1.4, in view of the observation that if $I$ is injective with regard to all monos (regular monos) then so is every internal power $I^{B}$. This observation follows from the right adjointness of $(-)^{B}$ to $B \times(-)$ and the fact that the latter functor preserves monos (regular monos).

We shall now consider a category $A$ which is an elementary topos, as defined by Lawvere and Tierney (see [10], [16], or [7]).

We assume a knowledge of their basic definitions, in particular of a to: pology $j$ in $A$ and the associated injective object $\Omega_{j}$, the equalizer of $j$ and $1_{\Omega}$. (See, e.g., [7, pp. 33-34].)

PROPOSITION 2.2. Let $\mathrm{A}$ be an elementary topos.

(i) If $I$ is an injective in $A$, the associated reflector $A \rightarrow$ Fix $Q$ preserves finite limits.

(ii) If $j$ is a topology in $A$, the reflective subcategory Fix $Q$ determined by the injective $\Omega_{j}$ consists of the $j$-sheaves.

Proof. (i) We know from Proposition 2.1 that Fix $Q=L\left(\left\{I^{A} \mid A \in A\right\}\right)$ and that $Q$ preserves monos (all monos in a topos are regular). Thus, noting that $\left(I^{A}\right)^{A^{\prime}} \cong I^{A \times A^{\prime}}$, we need only prove 
LEMMA 2.3. Let $A$ be an elementary topos and $J$ a class of injectives of A such that $I^{A}$ is in $J$ for all $I$ in $J$ and $A$ in $A$. Suppose the limit closure $L(J)$ of $J$ is a reflective subcategory and the reflector preserves monos. Then the reflector preserves finite limits.

Proof. (If $A$ is complete, the supposition about the reflector follows from [3], [14] or [15] .) Since the functor $(-)^{A}: A \rightarrow A$ is a right adjoint, it preserves limits. Since $L(J)$ is limit closed, the class of all objects $B$ of $A$ such that $B^{A}$ is in $L(J)$ is also limit closed. It follows from the assumption of the lemma that this class contains $J$, hence $L(J)$ and therefore:

$$
B^{A} \text { is in } L(J) \text { for all } B \text { in } L(J) \text { and } A \text { in } A .
$$

The rest of the proof of the lemma is obtained by putting together some propositions in [7]. We outline the proof, referring to [7] at several points.

In view of [7, Proposition 1.31], it follows from (*) that $R$ preserves finite products. It remains to show that it preserves equalizers. The equalizer of $f, g: A \longrightarrow A^{\prime}$ is the pullback of $A \stackrel{\langle 1, f\rangle}{\longrightarrow} A \times A^{\prime} \stackrel{\langle 1, g\rangle}{\stackrel{1}{\longrightarrow}} A$, where $\langle 1, f\rangle$ and $\langle 1, g\rangle$ are monos. Thus, we need only show that $R$ carries all pullbacks

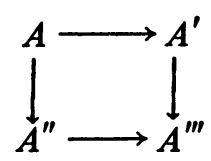

of $A$ in which all four maps are monos to pullbacks of $L(J)$.

If $I$ is an injective of $A$, then the following is a pushout in Sets [7, Corollary 2.55]:

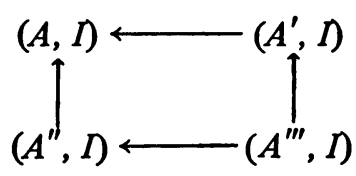

If $I$ lies in $J \subseteq L(J)$,then by adjointness we obtain the following pushout:

Suppose now that
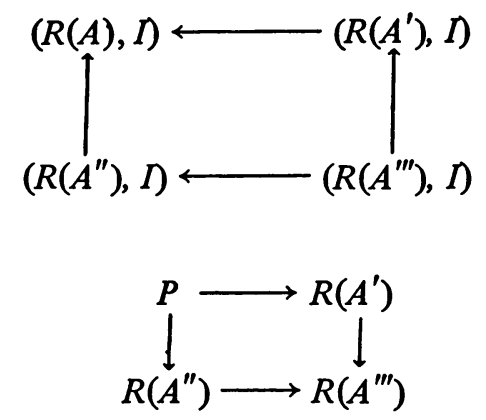
is a pullback in $L(J)$, hence also in A. Since $R$ preserves monos, all maps in this pullback are monos of $L(J)$, hence of $A$. Thus if we apply $(-, I)$, we again get a pushout in Sets. We certainly have a map $f: R(A) \rightarrow P$ such that the two triangles in the following diagram commute:

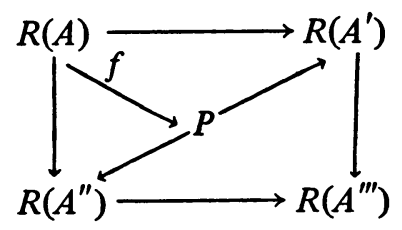

We want to show that $f$ is an isomorphism. In view of the two pushouts we know that $(f, I):(P, I) \rightarrow(R(A), I)$ is a bijection for any $I$ in $J$.

Now it is easily seen that the class of all objects $B$ of $A$ such that $(f, B)$ is a bijection is closed under formation of limits in $A$. As we have just shown, this class contains $J$, hence it contains $L(J)$. In particular, $(f, R(A))$ is a bijection, and therefore $f$ has a left inverse. Since $(f, B)$ is one-to-one for each $B$ in $L(J)$, $f$ is clearly an epi of $L(J)$, hence it is an isomorphism. The proof of the lemma is now complete.

We turn to the proof of Proposition 2.2(ii). Note that $\Omega_{j}$ is injective, because it is a retract of the injective $\Omega$. The fact that Fix $Q$ is the set of $j$-sheaves follows from these assertions:

(1) $\Omega_{j}$ is a sheaf.

(2) Every internal power of a sheaf is a sheaf.

(3) Every limit of sheaves is a sheaf.

(4) If $B$ is a sheaf, then there is a mono $B \rightarrow \Omega_{j}^{B}$ obtained from the (closed) diagonal map $B \rightarrow B \times B$.

(5) Every mono between two sheaves is closed, hence is a pullback of a $\operatorname{map} 1 \rightarrow \Omega_{j}$.

Proofs of these assertions not using the reflector can be found in the references, except for (3), which is trivial. It follows from (1) to (3) that all objects in Fix $Q$ are sheaves, and from (4) and (5) that every sheaf is in Fix $Q$, as the terminal object 1 is in Fix $Q$.

Lawvere and Tierney showed that if $A$ is an elementary topos and $R$ : $A \rightarrow B$ a reflector preserving finite limits, then $B$ is the category of $j$-sheaves for some topology $j$ in A.

3. Sheaves from a single injective. We now prove two results announced in [9].

Let $X$ be a small category and $A$ the topos of all functors $X^{\text {op }} \rightarrow$ Sets. We asserted in [9, Example 4] that any sheaf subcategory of $A$ is the limit 
closure of a single injective. A sheaf subcategory is well known to be the limit closure of a class of injectives, as discussed in $\S 2$.

Let us ask quite generally when is the limit closure of a class of injectives already the limit closure of a single injective, so that a reflector can be obtained by the construction of [9]? This is well known to be true in a module category Mod $R$. It is not true in the category of uniform spaces [9, Example 3]. We will show that it is true in functor categories.

PROPOSITION 3.1. Let A be a category satisfying the following conditions:

(a) A is co-well-powered and complete and has cokernel pairs.

(b) A has a generating set $G$.

(c) Every mono in $A$ is regular.

(d) If $I$ and $J$ are injectives in $A$ then there is a map $I \rightarrow J$.

Then the limit closure $L(J)$ of any class $J$ of injectives of $A$ is the limit closure $L(I)$ of a single injective $I$.

REMARK. Not all the above assumptions are necessary. However, they are satisfied when $A$ is the category of presheaves. Indeed, the set of representable functors $(-, X): X^{\text {op }} \rightarrow$ Sets is a generating set, and property (d) follows from the fact that $O \rightarrow I$ is mono. A module category $\operatorname{Mod} R$ also satisfies the above conditions.

Proof. A well-known consequence of (a) and (c) is the fact that every map in $A$ can be factored uniquely (up to isomorphism) as an epi followed by a mono. In view of $[14$, Theorem 5$], L(J)$ is therefore a reflective subcategory of $A$ and the reflector preserves regular monos, which means in this case that it takes monos of $A$ to regular monos of $L(J)$. (This also follows from [3] or [15].)

As an immediate consequence we observe that the category $L(J)$ also has the property that every mono is regular. In fact, $L(J)$ satisfies all the conditions (a) to (d). The generating set of $L(J)$ is obtained as the set of reflections of the generating set of $A$. The properties in condition (a) are obvious, except that $L(J)$ is co-well-powered. To see this, suppose $f: A \rightarrow B$ is an epi in $L(J)$. Then there is an epi $g: A \rightarrow C$ in A such that $h: C \rightarrow B$ is the best approximation of $C$ in $L(J)$ and $f=h g$.

We recall from [14, Theorem 1] that $L(J)$ has injective extensions, that is, each object is a regular subobject of an injective.

It will follow from Lemma 3.2 below that $L(J)$ has an injective cogenerator I. Since all monos are regular, each object of $L(J)$ is the equalizer of a pair of maps $I^{m} \Longrightarrow I^{n}$ and so $L(J)$ is the limit closure of $I$.

LEMMA 3.2. If a category B satisfies conditions (a)-(d) and has injective extensions, then it has an injective cogenerator. 
Proof. As already pointed out, it follows from (a) and (c) that every map in $B$ has an epi-mono factorization. Let $B$ be an object of $B, G \in G$, and $a, b \in(G, B)$, then the map $G+G \stackrel{[a, b]}{\longrightarrow} B$ factors as

$$
G+G \stackrel{e(a, b)}{\longrightarrow} K(a, b) \stackrel{m(a, b)}{\longrightarrow} B
$$

where $e(a, b)$ is epi and $m(a, b)$ is mono. Let $I(a, b)$ be an injective extension of $K(a, b)$ with monomorphism $i(a, b): K(a, b) \rightarrow I(a, b)$. Then there is a map $f(a, b): B \rightarrow I(a, b)$ such that $f(a, b) m(a, b)=i(a, b)$.

We claim that the set of injectives $I(a, b)$ cogenerates $B$. Indeed, suppose two maps into $B$ are equalized by all maps of $B$ into any element of this set. We want to show that these two maps are equal. Since $G$ generates $B$, we may assume, without loss in generality, that the two maps have the form $a, b: G \rightarrow$ $B$, where $G \in G$, hence that $f(a, b) a=f(a, b) b$. Now $a=m(a, b) e(a, b) k_{1}$ and $b=m(a, b) e(a, b) k_{2}$, where $k_{1}, k_{2}: G \rightarrow G+G$ are the two canonical injections. Therefore, $i(a, b) e(a, b) k_{1}=i(a, b) e(a, b) k_{2}$, hence $e(a, b) k_{1}=e(a, b) k_{2}$, and so $a=b$.

Now condition (d) implies that the product of a cogenerating set of injectives is a cogenerator, and it is obviously injective. This completes the proof of the lemma.

We recall that the limit closure of a class of injectives has been called a TFD subcategory in [14].

COROLLARY 3.3. Any TFD subcategory of the category of all functors $X^{\mathrm{op}} \rightarrow$ Sets is the limit closure of a single injective.

In a preprint of a forthcoming paper [12, Propositions 5.10, 5.17] Meyer obtains a similar result.

The reader is warned that the reflector onto a TFD subcategory of a functor category need not preserve finite products [3, Example 7.4].

We are now in a position to prove the assertion in [9, Example 7].

PROPOSITION 3.4. Let $X$ be any small category, $B$ a TFD subcategory of the category $A$ of all functors $X^{\mathrm{op}} \rightarrow$ Sets. Then $B^{\mathrm{op}}$ is varietal over Sets.

REMARK. The special case of this proposition with $B=A$ was obtained by Bunge [4].

Proof. We apply [9, Proposition 3]. It is clear that $B$ is complete and has cokernel pairs. We have shown that it has an injective cogenerator. This is a "regular" cogenerator in the sense of [9, Proposition 2], because all monos in $B$ are regular.

It remains to show that any coequivalence relation 


$$
B \underset{v}{\stackrel{u}{\longrightarrow}} D
$$

is a cokernel pair. Now it follows from the definition of "equivalence relation" in $B^{\circ p}$ given in [9] that there is a map $d: D \rightarrow B$ such that $d u=1_{B}=$ $d v$. Let $k: K \rightarrow B$ be the equalizer of the pair $(u, v)$,

$$
B \underset{v^{\prime}}{\stackrel{u^{\prime}}{\rightleftharpoons}} C
$$

the cokernel pair of $k$ in $A$, and $h: C \rightarrow D$ the unique map such that $h u^{\prime}=u$ and $h v^{\prime}=v$.

We claim that $h$ is a monomorphism in $A$. This will follow if we show that $h(X): C(X) \rightarrow D(X)$ is mono in Sets for each $X$ in $X$. Indeed, $C(X)$ is the disjoint union of two copies of $B(X)$ with the image of $k(X)$ amalgamated, and $u^{\prime}(X), v^{\prime}(X)$ are the two inclusions $B(X) \Rightarrow C(X)$. Suppose $x, y \in C(X)$ and $h(X)(x)=h(X)(y)$. If $x$ and $y$ lie in different copies of $B(X)$, then $x=u^{\prime}(X)(z)$ and $y=v^{\prime}(X)(t)$ for some $z, t \in B(X)$. Thus $u(X)(z)=v(X)(t)$, but $d(X) u(X)=$ $1=d(X) v(X)$, hence $z=t$, and $z \in \operatorname{Im} k(X)$, and therefore $x=y$. If $x$ and $y$ lie in the same copy of $B(X)$, we proceed similarly.

Thus $h$ is a regular mono in $A$. Let $R: A \rightarrow B$ be the reflector and consider

$$
K \stackrel{k}{\longrightarrow} B=R(B) \stackrel{R\left(u^{\prime}\right)}{R\left(v^{\prime}\right)} R(C) \stackrel{R(h)}{\longrightarrow} R(D) .
$$

Here $\left(R\left(u^{\prime}\right), R\left(v^{\prime}\right)\right)$ is the cokernel pair of $R(k)=k$ in $B$. Since the reflector preserves regular monos, $R(h)$ is a regular mono in $B$. But $R(h) R\left(u^{\prime}\right)=R(u)=$ $u$ and $R(h) R\left(v^{\prime}\right)=R(v)=v$, and the coequivalence relation $(u, v)$ is jointly epi. Therefore, $R(h)$ is epi in $B$. Being also a regular mono, $R(h)$ is an isomorphism, hence $(u, v)$ is the cokernel pair of $k$.

4. Further applications to topos theory. We shall give another description of the localization functor $Q$ which has useful consequences. As in $\S 1, Q$ is obtained by Fakir's process from the triple $S=U F$, where $A \stackrel{F}{\longrightarrow} B \stackrel{U}{\longrightarrow} A$ is a pair of functors with $F$ left adjoint to $U$. Let $B_{C}$ be the category of coalgebras of the cotriple $C=F U$ on $B$. The comparison functor $K: A \rightarrow B_{C}$ and its right adjoint $M$ are defined in the usual way [11, p. 135]. It is easy to check that $M K \cong Q$, as we observed in [9, Proposition 1] in a special case (see also [2]).

Denote the inclusion of Fix $Q$ into $A$ by $I$. We always have $\operatorname{Im} U \subseteq$ Fix $Q$, so $U$ factors as $I U^{\prime}$, and $U^{\prime}$ has left adjoint $F^{\prime}=F I$ with $F^{\prime} U^{\prime}=F U=$ $C$. The comparison functor Fix $Q \rightarrow B_{C}$ induced by the pair $\left(F^{\prime}, U^{\prime}\right)$ is 
$K^{\prime}=K I$. If $Q$ is idempotent then Fix $Q$ is limit closed in A, by Proposition 1.2, hence $M$ factors through Fix $Q$ as $I M^{\prime} . M^{\prime}$ has left adjoint $K^{\prime}$, and $I M^{\prime} K^{\prime}=$ $M K I \cong Q I=I$, hence $M^{\prime} K^{\prime}$ is isomorphic to the identity on Fix $Q$. Thus $K^{\prime}$ embeds Fix $Q$ into $B_{C}$ as a coreflective full subcategory. We summarize our conclusions:

PROPOSITION 4.1. If $Q$ is idempotent, the pair $(F, U)$ can be factored thus:

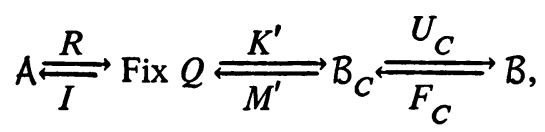

where $F \cong U_{C} K^{\prime} R, U \cong I M^{\prime} F_{C}$. Here

$$
\begin{aligned}
I & =\text { inclusion }, & R & =\text { reflector }, \\
M^{\prime} & =\text { coreflector }, & K^{\prime} & =\text { full embedding, } \\
F_{C} & =\text { cofree functor }, & U_{C} & =\text { forgetful functor } .
\end{aligned}
$$

Proof. $\left({ }^{3}\right)$ It remains to check the two isomorphisms. Certainly $I M^{\prime} F_{C}=$ $M F_{C} \cong U$, taking adjoints of $U_{C} K=F$. Again, by taking adjoints, we obtain $U_{C} K^{\prime} R \cong F$. Alternatively, one may calculate $U_{C} K^{\prime} R=F I R=F Q \cong F$, since $F\left(\eta_{1}(A)\right)$ is an isomorphism for each object $A$ of $A$. This is because, for any map $a: A \rightarrow A^{\prime}$ in A, $F(a)$ is an isomorphism if and only if $Q(a)$ is one (by an argument like that for Lemma 1.3).

Corollary 4.2. If $Q$ is idempotent, $F^{\prime}$ creates colimits. Thus Fix $Q$ has all colimits that $B$ has.

Proof. It is well known that the forgetful functor $U_{C}: B_{C} \rightarrow B$ creates colimits. Moreover, so does $K^{\prime}$ : Fix $Q \rightarrow B_{C}$, as a coreflective subcategory is closed under colimits. Hence $F^{\prime}=U_{C} K^{\prime}$ creates colimits.

REMARK. In fact, it is clear that colimits in Fix $Q$ may be calculated as follows: Let $\Gamma: X \rightarrow$ Fix $Q$ be any diagram and assume that $F^{\prime} \Gamma$ has a colimit. Then so does $K^{\prime} \Gamma$, and colim $\Gamma=M^{\prime}\left(\operatorname{colim} K^{\prime} \Gamma\right)$.

Our factorization generalizes the factorization of geometric morphisms between (elementary) toposes. In this special case [8, Theorem 4.3], $A$ and $B$ are toposes and $F$ preserves finite limits, moreover $K^{\prime}$ is an equivalence with inverse $M^{\prime}$, so that $F^{\prime}$ is cotripleable.

(3) (ADDED JANUARY, 1975). A similar factorization, in the case when $U F$ and $F U$ are idempotent, has been considered by J. Isbell. See Top and its adjoint relatives, General Topology and its Relations to Modern Analysis and Algebra (Proc. Conf., Kanpur 1968), Academic Press, 1971, pp. 149-152. 
What conditions on $A, B$ and $F$ will assure that $F^{\prime}$ is cotripleable? We know that $F^{\prime}=U_{C} K^{\prime}$ reflects isomorphisms, since both $U_{C}$ and $K^{\prime}$ do. By Beck's crude cotripleability theorem [11], $F^{\prime}$ will certainly be cotripleable if it preserves equalizer diagrams. We obtain a better result using Duskin's conditions [5, Theorem 3.2].

Proposition 4.3. Suppose $B$ has cokernel pairs and $Q$ is idempotent. Then $F^{\prime}$ is cotripleable if and only if it preserves equalizers of coequivalence relations in Fix $Q$ whose images under $F^{\prime}$ are cocontractible.

Proof. Since, by Corollary 4.2, $F^{\prime}$ creates colimits, Duskin's condition (b) is always satisfied. Since $F^{\prime}$ reflects isomorphisms, his condition (a) reduces to the condition of the proposition.

We now apply the above results to obtain new proofs $\left({ }^{4}\right)$ of two theorems due to Mikkelsen [13].

PROPOSITION 4.4. Let $E$ be a cartesian closed category with finite limits and a subobject classifier $\Omega$. Then $E$ has finite colimits, hence is a topos.

Proof. It is easily shown that $\Omega$ is injective. Moreover, for any object $E$ of $E$, the singleton map $E \rightarrow \Omega^{E}$ is a monomorphism, hence there is an equalizer diagram $E \rightarrow \Omega^{E} \Longrightarrow \Omega$. By Proposition 2.1, the localization functor $Q$ determined by the adjoint pair

$$
E \stackrel{\Omega^{(-)}}{\longrightarrow} E^{\mathrm{op}} \stackrel{\Omega^{(-)}}{\longrightarrow} E
$$

is the identity functor on $E$, that is, Fix $Q=E$. By Corollary 4.2, $E$ has all colimits which $E^{\mathrm{op}}$ has, hence all finite colimits.

In view of the remark following Corollary 4.2 , we can calculate finite colimits in $E$ explicitly in terms of the corresponding limits, powers of $\Omega$ and equalizers (used in defining $M^{\prime}$ ). For example, the coequalizer of the pair of maps $f, g: A \rightarrow B$ can be found as follows.

Let $S(A)=\Omega^{\Omega A}$, as before. Let $m: C \rightarrow \Omega^{B}$ be the equalizer of $\left(\Omega^{f}, \Omega^{g}\right)$. There is a unique map $t: S(C) \rightarrow C$ such that $m t=\Omega^{\eta(B)} S(m)$. In fact, $(C, t)$ is a coalgebra in $\left(E^{o p}\right)_{S}$ and is the codomain of the coequalizer of $\left(K^{\prime}(f), K^{\prime}(g)\right)$. Now let $u: E \rightarrow \Omega^{C}$ be the equalizer of $\left(\eta\left(\Omega^{C}\right), \Omega^{t}\right): \Omega^{C} \Rightarrow$ $S\left(\Omega^{C}\right)$. Then $E=M^{\prime}(C, t)$. There is a unique map $v: B \rightarrow E$ such that $u v=\Omega^{m} \eta(B)$. This map $v$ is $M^{\prime}\left(\operatorname{coeq}\left(K^{\prime}(f), K^{\prime}(g)\right)\right)$, hence is coeq $(f, g)$ in $E$.

(4) (ADDED JANUARY, 1975). These theorems have also been proved by R. Paré. His proof has appeared in Colimits in topoi, Bull. Amer. Math. Soc. 80 (1974), 556-561. 

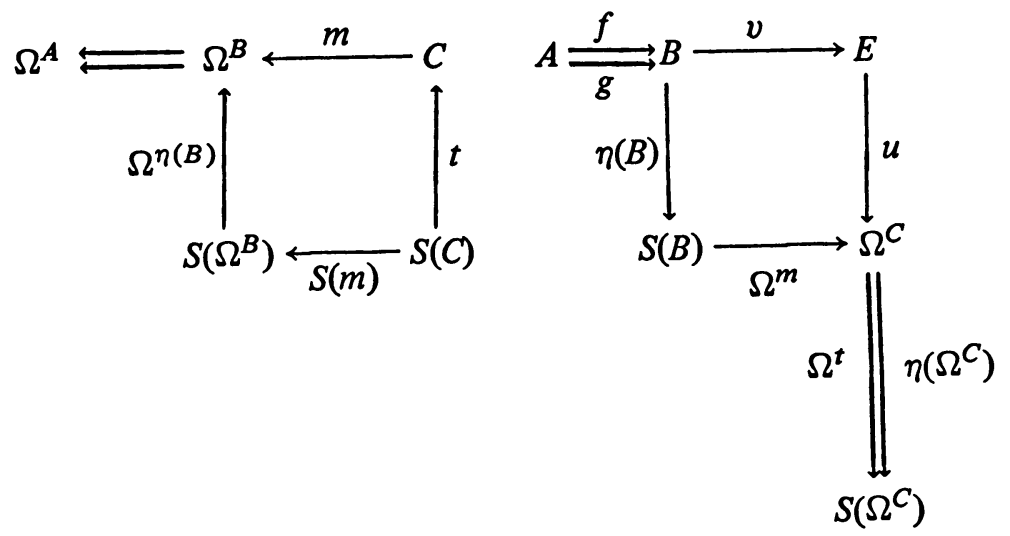

It follows from the existence of such explicit constructions that any functor between toposes which preserves exponentiation, the subobject classifier and finite limits also preserves finite colimits.

Proposition 4.5. Any topos $E$ is cotripleable over $E^{\mathrm{op}}$, that is, $E^{\mathrm{op}}$ is tripleable over $E$.

Proof. Mikkelsen [13] has shown that every coequivalence relation in $E$ is the cokernel pair of its equalizer. The functor $F=\Omega^{(-)}: E \rightarrow E^{o p}$ preserves regular monos, by Proposition 2.1, and it preserves cokernel pairs, as it has a right adjoint. Thus it preserves equalizer diagrams

$$
A \stackrel{m}{\longrightarrow} B \underset{g}{\stackrel{f}{\longrightarrow}} C
$$

in which $(f, g)=$ cok $m$. By Proposition $1.8, F$ is cotripleable.

\section{REFERENCES}

1. H. Applegate and M. Tierney, Categories with models, Sem. on Triples and Categorical Homology Theory (ETH, Zürich, 1966/67), Lecture Notes in Math., vol. 80, SpringerVerlag, Berlin, 1969, pp. 156-244. MR 39 \#4243.

2. - Iterated cotriples, Reports of the Midwest Category Seminar. IV, Lecture Notes in Math., vol. 137, Springer-Verlag, Berlin, 1970, pp. 56-99. MR 42 \#338.

3. M. Barr, Non-abelian torsion theories, Canad. J. Math. 25 (1973), 1224-1237.

4. M. C. Bunge, Relative functor categories and categories of algebras, J. Algebra 11 (1969), 64-101. MR 38 \#4536.

5. J. Duskin, Variations on Beck's tripleability criterion, Reports of the Midwest Category Seminar, III, Lecture Notes in Math., vol. 106, Springer-Verlag, Berlin, 1969, pp. 74-129. MR 40 \#5691.

6. S. Fakir, Monade idempotente associée à une monade, C. R. Acad. Sci. Paris Sér. A-B 270 (1970), A99-A101. MR 41 \#1828.

7. P. Freyd, Aspects of topoi, Bull. Austral. Math. Soc. 7 (1972), 1-76.

8. A. Kock and C. G. Wraith, Elementary toposes, Lecture Notes Series 30, Aarhus University, 1971. 
9. J. Lambek and B. Rattray, Localization at injectives in complete categories, Proc. Amer. Math. Soc. 41 (1973), 1 -9.

10. F. W. Lawvere, Quantifiers and sheaves, Proc. Internat. Congress Math. (Nice, 1970), vol. 1, Gauthier-Villars, Paris, 1971, pp. 329-334.

11. S. Mac Lane, Categories for the working mathematician, Springer-Verlag, New York, 1971.

12. H.-M. Meyer, Spektraltopoi, Universität Tübingen, 1972 (preprint).

13. C. J. Mikkelsen, Internal completeness of elementary topoi, Thesis, Aarhus University, 1973.

14. B. Rattray, Non-additive torsion theories, Manuscripta Math. 12 (1974), 285-305.

15. C. M. Ringel, Monofunctors as reflectors, Trans. Amer. Math. Soc. 161 (1971), 293-306. MR 45 \#1989.

16. M. Tierney, Axiomatic sheaf theory, Bull. Amer. Math. Soc. (to appear).

17. F. Ulmer, On modules and objects which are flat over their endomorphism rings, Universität Zürich, 1971/72 (preprint).

18. - On the existence and exactness of the associated sheaf functor, J. Pure Appl. Algebra 3 (1973), 295-306.

19. D. H. Van Osdol, Sheaves in regular categories, Lecture Notes in Math., vol. 236, Springer-Verlag, New York, 1971, pp. 223-239. CANADA

DEPARTMENT OF MATHEMATICS, MCGILL UNIVERSITY, MONTREAL, QUEBEC, 